
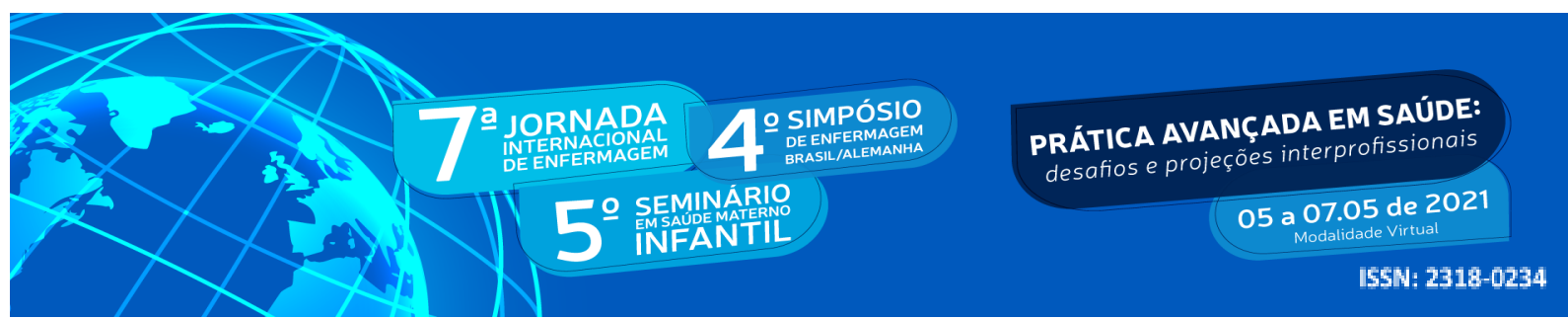

DOI: http://doi.org/10.48195/jie2021-051

\title{
PRÁTICAS AVANÇADAS EM SAÚDE SOB A LUZ DAS TEORIAS E SISTEMATIZAÇÃO DA ENFERMAGEM
}

\begin{abstract}
Hélida Maravilha Dantas e Sousa Almeida ${ }^{1}$; Maria Fernanda Bandeira da Silva ${ }^{2}$; Mylena da Silva Maia ${ }^{3}$; Patrícia Adriana Silva ${ }^{4}$; Ana Elza Oliveira de Mendonça ${ }^{5}$
\end{abstract}

\begin{abstract}
RESUMO
O presente trabalho tem como objetivo refletir acerca do uso da sistematização da assistência de enfermagem e das teorias de enfermagem como subsídio as práticas avançadas em saúde. Trata-se de um estudo teórico reflexivo com abordagem qualitativa, realizado em janeiro de $2021 \mathrm{com}$ respaldo da literatura científica e experiências da prática clínica. Na análise dos dados foi observado que a prática avançada em saúde, juntamente com a sistematização da assistência de enfermagem e teorias de enfermagem proporcionam ao enfermeiro um manejo clínico diferenciado. Tal abordagem promove uma avaliação clínica, como também crítica e minuciosa, evitando uma assistência fragmentada e pautada no modelo biomédico. A abordagem integrativa, com maiores propriedades quanto a escolha da conduta para o cuidado a ser ofertado, favorece a autonomia e valorização profissional.
\end{abstract}

Palavras-chave: Práticas Avançadas em Saúde; Processo de Enfermagem; Teorias de Enfermagem.

\begin{abstract}
This paper aims to reflect on the use of systematization of nursing care and nursing theories as a subsidy to advanced health practices. This is a reflective theoretical study with a qualitative approach, carried out in January 2021 with the support of scientific literature and experiences of clinical practice. In the analysis of the data, it was observed that the advanced health practice, together with the systematization of nursing care and nursing theories provide the nurse with a differentiated clinical management. Such an approach promotes a clinical evaluation, as well as a critical and thorough evaluation, avoiding fragmented assistance based on the biomedical model. The integrative approach, with greater properties as to the choice of conduct for the care to be offered, favors autonomy and professional valorization.
\end{abstract}

Key Words: Health Advanced Practices; Nursing Process; Nursing Theories.

\footnotetext{
${ }^{1}$ Acadêmica de Enfermagem. Universidade Federal de Campina Grande E-mail: helidacaico@hotmail.com

${ }^{2}$ Acadêmica de Enfermagem. Universidade Federal de Campina Grande E-mail: fernanda2000bandeira@gmail.com

${ }^{3}$ Acadêmica de Enfermagem. Universidade do Estado do Rio Grande do Norte. E-mail: mylenamaia@alu.uern.br

${ }^{4}$ Acadêmica de Enfermagem. Universidade do Estado do Rio Grande do Norte. E-mail: patynascimento96@gmail.com

${ }^{5}$ Orientadora. Pós-Doutora em Enfermagem. Universidade Federal do Rio Grande do Norte. E-mail: anaelzaufrn@gmail.com
} 


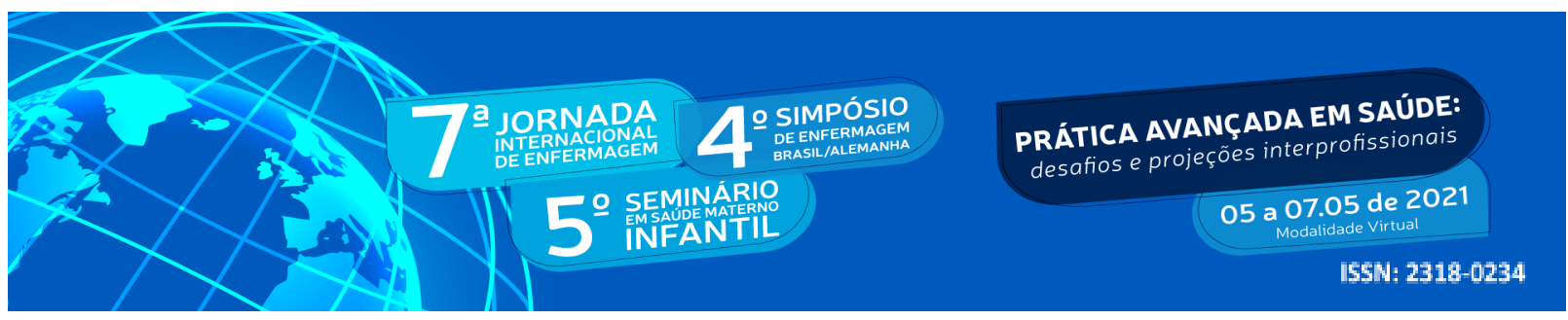

\section{INTRODUÇÃO}

A enfermagem é a ciência responsável pelo estudo, desenvolvimento e implementação do cuidado, e conta para o seu subsídio de teorias que fornecem respaldo à sua prática. Esse olhar científico teve seu advento com a pesquisadora Florence Nightingale, fundadora da Enfermagem moderna e da Teoria ambientalista, que proporcionou uma nova forma assistencial e operacional aos clientes durante a Guerra da Crimeia, resultando em uma notável redução dos óbitos. A partir desse marco, se observou a necessidade crescente de aliar a pesquisa com a prática cuidativa, vislumbrando maior segurança e qualidade aos pacientes (MARTINS; BENITO, 2016).

Além desse aspecto, foi pontuada a importância de sistematizar essa oferta de cuidado de forma a otimizar o trabalho da enfermagem, com eficiência e eficácia. Nesse contexto surgiu a Sistematização da Assistência de Enfermagem (SAE) que conta com vários instrumentos e métodos para tornar o cuidado mais organizado e estruturado. Uma forma de consolidar sua aplicação é através das cinco etapas presentes no Processo de Enfermagem (PE): Coleta de dados; Diagnóstico de Enfermagem; Planejamento; Implementação e Avaliação (GOMES et al., 2018).

Apesar de todo esse arsenal metodológico e científico que a Enfermagem possui, o interesse em adoção de práticas inovadoras em saúde vem ganhando destaque. Isso esta ocorrendo devido às mudanças de cenários que a saúde mundial vem passando, com o envelhecimento populacional, aumento de condições crônicas e da escassez de profissionais capacitados. Vislumbrando acompanhar tal realidade, surgiu a Enfermagem com Prática Avançada (EPA), que se constitui em um instrumento de inovação no fortalecimento do trabalho, aperfeiçoando a cobertura e acesso da população aos cuidados em saúde (BRYANT-LUKOSIUS et al., 2017; MIRANDA NETO et al., 2018).

Apesar dessa alternativa já existir a mais de 40 anos, sua implementação é mais presente em países desenvolvidos e que possuem maior renda, exigindo uma série de desafios na formação e desenvolvimento do enfermeiro para uma adesão a esse método. Entretanto, é inegável a importância desse artifício para o aprimoramento e destaque da assistência de 


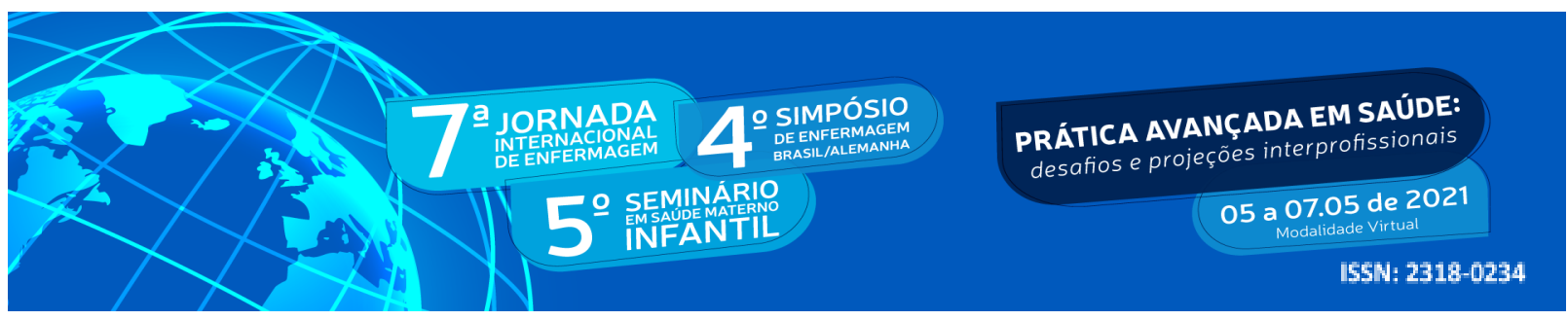

enfermagem na atualidade, como observado durante a pandemia do novo Coronavírus, que exigiu desse profissional maior autonomia em todas as esferas da saúde e enalteceu a necessidade de uma assistência integral ao cliente (SILVA et al., 2020).

Quando consolidado estas duas esferas do cuidado, a teoria aliada a SAE, é possível que o enfermeiro desenvolva uma assistência de qualidade e segurança, no campo da promoção, prevenção e assistência direta à saúde. Considerando a urgência de implementação das práticas avançadas, a reflexão sobre a adesão à EPA sob a luz das teorias e sistematização da assistência, faz-se fundamental configurando em um escopo indispensável na evolução do enfermeiro no campo da saúde.

\section{OBJETIVO}

Refletir acerca do uso da sistematização da assistência de enfermagem e das teorias de enfermagem como subsídio as práticas avançadas em saúde.

\section{METODOLOGIA}

A realização desse estudo adotou a abordagem qualitativa, utilizando a literatura científica como fonte de pesquisa para fundamentar essa obra. Para subsidiar a construção do tema e nortear a pesquisa, foi considerado o objetivo três da Agenda 2030 dos Objetivos de Desenvolvimento Sustentável (ODS), que é saúde e bem-estar. Essa metodologia, também desenvolvida por Almeida, Ramos e Ferreira (2020), consiste em um estudo teórico reflexivo que promove um olhar crítico sobre uma temática específica, conexa com a contemporaneidade.

Para guiar todo o processo de confecção deste estudo foi elaborada a seguinte questão: "como o uso da sistematização da assistência e das teorias de enfermagem subsidiam as práticas avançadas em saúde?”. A partir desse ponto, deu-se início a pesquisa bibliográfica, que ocorreu no mês de Janeiro de 2021 em bases de dados destinadas à indexação de artigos e periódicos científicos, como a Biblioteca Virtual em Saúde (BVS). Os descritores de assunto utilizados foram: "Health Advanced Practice"; "Advanced Practice Nursing"; "Nursing 


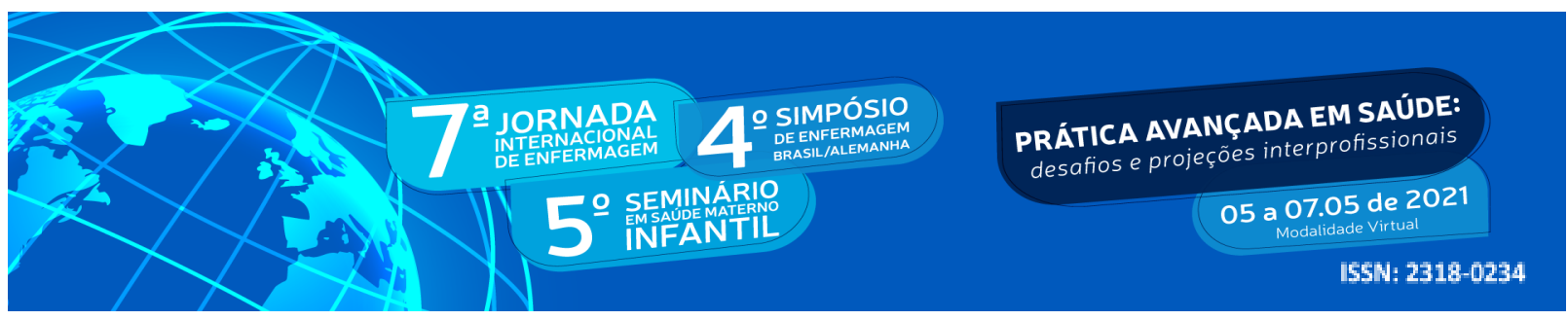

process"; "Nursing Theory". Estes termos foram pesquisados de forma individual e a partir de cruzamento com o uso do operador booleano " $A N D$ ".

Foram incluídos os estudos nos idiomas de português, inglês e espanhol, que contivesse em seu título ou resumo alguns dos termos chaves. Como critério de exclusão, foi adotado a duplicidade, a indisponibilidade na íntegra e que após a leitura na íntegra não apresentasse contribuição relevante para a discussão e para responder a questão norteadora da pesquisa. Foram priorizadas as obras em formato de artigo científico, publicadas nos últimos cinco anos, contudo, justica-se que foram incluídas bibliografias mais antigas devido possuirem grande relevância para a temática.

O material eleito foi analisado na íntegra, de forma individual, destacando os principais aspectos relacionados às Práticas Avançadas em Saúde compatíveis com o objetivo da investigação, e sendo refletidos sob a óptica das teorias e sistematização da enfermagem. Após essa etapa, deu-se início a construção dos resultados e discussões, objetivando a apresentação das informações obtidas e uma análise crítica e reflexiva do conteúdo, propondo uma alternativa acessível e aplicável com foco no empoderamento do enfermeiro para o desenvolvimento de uma atenção integral à saúde.

\section{RESULTADOS E DISCUSSÃO}

As práticas de saúde vêm passando por mudanças em todo o mundo, viabilizadas pelo avanço nas ciências da saúde, descobertas inovadoras de terapias e no surgimento de processos patológicos desafiadores, que obrigam os sistemas mundiais a adotarem uma nova forma de fornecer assistência à saúde para a população. Nesta conjuntura, a necessidade de adoção de um modelo de prestação de serviços integrais aos clientes ganha cada vez mais destaque e urgência, visto que hoje o ser humano é compreendido nas esferas biopsicossociais, e a saúde perpassa a definição de ausência de doenças (VITALI et al., 2019).

Uma estratégia que pode ser aplicada para facilitar a execução dessa nova abordagem são as práticas avançadas em saúde, sobretudo na área da Enfermagem. Esses profissionais são definidos pelo Conselho Internacional de Enfermagem (CIN, 2009) como o enfermeiro que possui conhecimento especializado, habilidades complexas para decisão e competência 


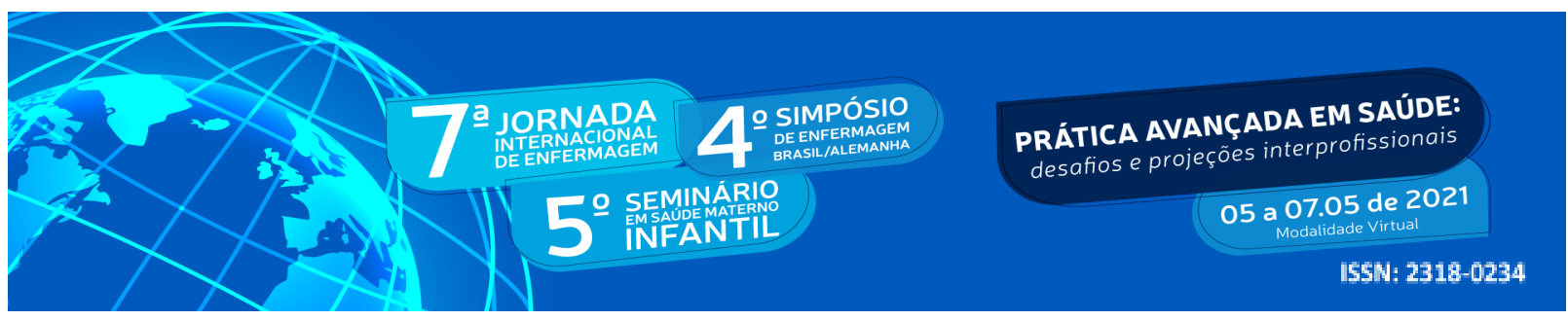

clínica expandida para a prática. Esses profissionais possuem dentre as suas atividades, a capacidade de diagnosticar clinicamente um paciente, prescrever medicamentos independentemente de controle médico e indicar tratamentos médicos, referenciar e contra referenciar.

São apontados alguns títulos para referir esse profissional, sendo os mais conhecidos "Nurse practitioner" que diagnostica, prescreve medicamentos e realiza procedimentos por meio de experiências adquiridas no cotidiano; e o "Clinical Nurse Specialist" que possui especializações em Enfermagem Clinica, com título de mestre ou doutor, toma decisões complexas e atua em gestão e educação em saúde. Entretanto, não há um total conhecimento dos escopos desses profissionais, visto que variam os padrões de prática dependendo do país. Isso ocorre devido a regulamentação desse profissional variar de acordo com a nação, visto as necessidades específicas daquela população, todavia, isto dificulta a implementação dessa classe por outros países (BRYANT-LUKOSIUS et al., 2017; MIRANDA NETO et al., 2018).

Ainda, para o enfermeiro receber este título, são necessários alguns pré-requisitos além da normatização do Estado, como a presença de pós-graduação podendo ser residências e mestrado profissional, experiências em práticas avançadas em saúde, além de possuir um senso de liderança, coordenação, pesquisa, e tomadas de decisões. Nesse contexto, há três bases para essa implementação: definição dos papéis, regulamentação e formação (PIMENTA, 2018).

Toda essa autonomia necessária à função do EPA é uma pauta que é desenvolvida com o surgimento da Enfermagem moderna. Essa capacidade e coordenação do cuidado facilita no desenvolvimento de ações assertivas, auxiliando ainda no olhar holístico para o paciente. As teorias de enfermagem oferecem esse respaldo científico ao profissional, garantindo ainda, maior independência e segurança na sua prática assistencial. Barbosa e Silva (2018) identificaram que as teóricas mais referenciadas no Brasil, na elaboração da SAE foram a "Teoria da Adaptação" de Callista Roy e a "Teoria do cuidado Transpessoal” de Jean Watson.

Ainda pode ser mencionado como fundamentação científica para o estabelecimento de uma assistência integral aos pacientes a "Teoria das Necessidades Humanas Básicas" de Wanda Horta e as "Teorias do Autocuidado" de Dorothea Orem. A relevância dessas pesquisadoras no âmbito da enfermagem é mais destacado quando avaliado a atual situação 


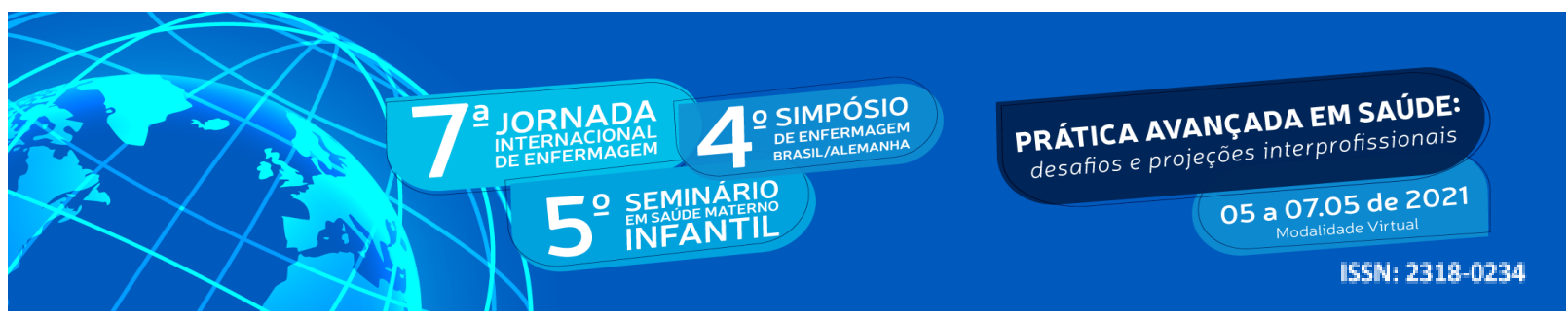

mundial, visto que esse olhar integral proporcionado por essas teorias auxiliam na identificação de déficits na saúde dos seus pacientes, transcendendo o olhar sob os diagnósticos já esperados devido ao aumento das doenças crônicas (BRANDÃO, et al., 2019). Quando considerado a pandemia e o surgimento das infecções resistentes aos tratamentos, há urgência em uma intervenção no processo saúde/doença sob a perspectiva biopsicossocial, integral e multidisciplinar, e esse arcabouço teórico pode conceber uma inovação no manejo desses problemas.

Apesar de importante, foi evidenciado por Alcântara et al. (2011) a identificação da baixa adesão e consequentemente, a necessidade do enfermeiro elencar uma teoria de enfermagem antes da implementação da SAE, para esta ter uma maior eficácia. Essa realidade ainda foi identificada em um estudo mais recente, que exaltando uma baixa utilização desse artifício científico junto com o processo de enfermagem, apesar de sua evidente importância, visto que a teoria de enfermagem contribui para a reflexividade e criticidade baseada na ciência e estruturadas a partir de 4 conceitos maiores, são eles o humano, saúde, meio ambiente e enfermagem (BARBOSA; SILVA, 2018).

Esse processo de adesão à uma teoria de enfermagem nas práticas cuidativas pode ser um grande aliado ao contexto do EPA, visto que este profissional terá um diferencial no manejo clínico, agregando às competências adquiridas com a capacitação das práticas avançadas. Dessa forma, conseguirá não somente agir clinicamente, mas também avaliar de forma crítica e cuidadosa os déficits relacionados ao cuidado em saúde. Sousa (2016) expõe a possibilidade sobre o uso da Teoria das Transições de Meleis junto dessa realidade, evitando que esse profissional se resuma em um enfermeiro sustentado pelo modelo biomédico.

Essa teoria afirma que cada período de transformação acarreta novos comportamentos e redefine o indivíduo, dessa forma, promove mudanças em todo o contexto do ser incluindo a saúde. Devido a sua estrutura ampla, possibilita a identificação das vulnerabilidades e durante essas transições permitindo a realização de intervenções específicas (MELEIS, 2010). Todavia, há um vasto depósito de doutrinas que podem ser associadas às práticas avançadas e que podem conceder uma maior individualidade ao serviço prestado.

Ilustrando melhor essa possibilidade de relação mútua entre esses aspectos, pode ser cogitado o seguinte cenário: a realização da SAE na prática do enfermeiro de prática 


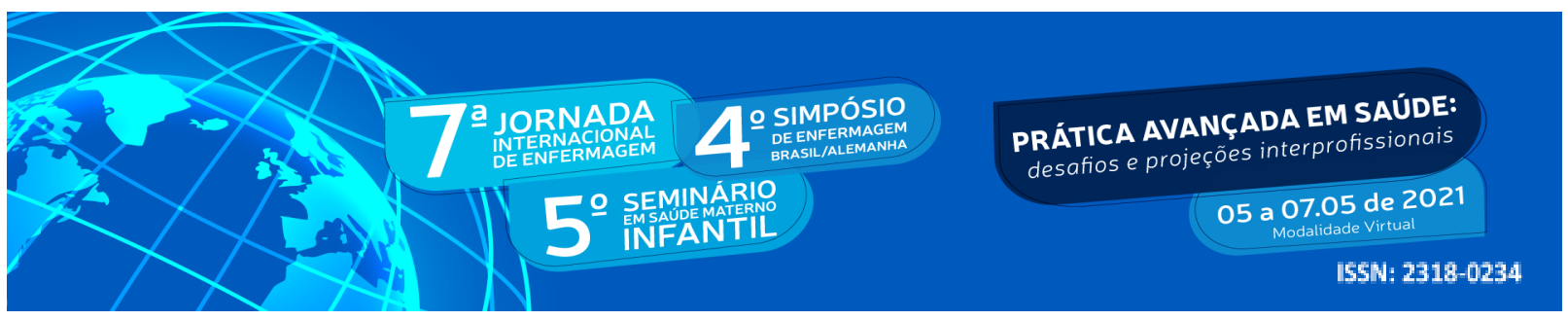

avançada, através do processo de enfermagem subsidiado por um teórico, em que este profissional não apenas realiza sua atividade clínica, mas também atua na prescrição crítica e integral do cuidado, realizado sistematicamente.

Este panorama pode ser refletido frente às sete práticas clínicas que o Conselho Internacional de Enfermagem (2009) aponta para o EPA: 1. Autonomia para prescrever (qualquer medicamento); 2. Autonomia para solicitar exames; 3. Autonomia para diagnosticar e avaliar de forma clínica (não inclui o diagnóstico de enfermagem); 4. Autonomia para indicar tratamento médico (não incluí as atividades já reconhecidas pela enfermagem); 5. Responsabilidade sobre um conjunto de usuários específicos; 6. Autonomia para referenciar e contrarreferenciar clientes; 7. Profissional de referência para primeiro contato.

Ao observar tais atividades elencadas, é possível perceber a ênfase designada para a "autonomia" profissional. Mas, para que isso ocorra, é preciso confiança e segurança, especialmente para tomar decisões, e é aqui que o cenário anterior se enquadra. A utilização das cinco etapas do Processo de Enfermagem, atrelada a uma avaliação e diagnóstico clínico, juntamente com a confecção de diagnósticos de enfermagem e um plano de cuidado embasado por uma teórica da área, concebe uma abordagem em saúde de forma integral, como demanda a atualidade, e pode funcionar como uma estratégia facilitadora nas decisões clínicas que deverão ser tomadas.

Além disso, esta tática fornece destaque ao EPA, evitando que este se limite ao modelo biomédico, totalmente focado no processo patológico e no tratamento específico ao problema clínico diagnosticado (SOUSA, 2016). Como também promover a maior valorização e destaque da enfermagem, tanto pelo próprio profissional, que irá incrementar o seu processo de trabalho com as técnicas elaboradas por essa ciência, que ainda é pouco difundida, como pela sociedade, que terá uma prestação de serviço de saúde de maior qualidade, segurança, integralidade e multidimensionalidade.

\section{CONCLUSÃO}

A reflexão sobre as práticas da enfermagem é um assunto relevante e que ganha cada vez mais notoriedade com o advento da pandemia da COVID-19. As discussões mundiais 


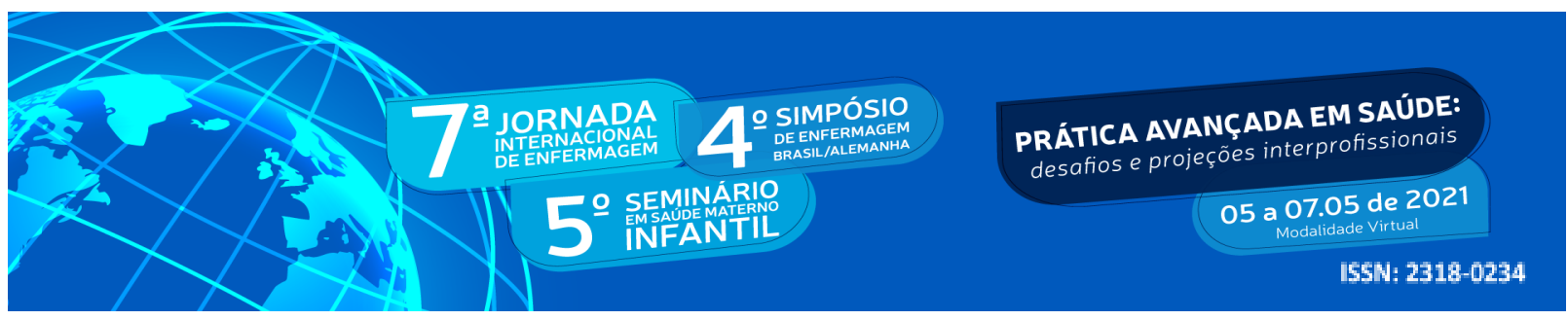

acerca da implementação das Práticas Avançadas em Enfermagem, abriram espaço para conjecturas acerca dessas condutas associadas aos métodos já instituídos nesta ciência, para uma melhor oferta assistencial ao paciente. A pesquisa revela a necessidade do uso das teorias associadas ao processo de enfermagem como alternativa facilitadora das práticas deste profissional, vislumbrando uma maior autonomia e evitando atitudes plenamente focadas na doença, que descaracterizaria o cuidado como integral. Foi identificada uma limitação na produção científica que relacione as práticas avançadas com a SAE e teorias de enfermagem, sendo requeridos novos estudos que busquem relacionar melhor essas esferas táticas.

\section{REFERÊNCIAS}

ALCÂNTARA, M. R. ET AL. Teorias de Enfermagem: A Importância Para A Implementação Da Sistematização Da Assistência De Enfermagem. Rev Cien Faema, Ariquemes, v. 2, n. 2, p. 115-132, out. 2011. [Citado em 17 Março 2021]. Disponível em: http://www.faema.edu.br/revistas/index.php/Revista-FAEMA/article/view/99.

ALMEIDA, H. M. D. S.; RAMOS, A. C. A.; FERREIRA, S. B. Fitoterapia E Saúde Mental: Estudo À Luz Da Teoria De Orem. Revista Interdisciplinar em Saúde, Cajazeiras, 7 (único): 482-496, 2020 [Citado em 15 Março 2021]. Disponível em: http://www.interdisciplinaremsaude.com.br/Volume_28/Trabalho_37_2020.pdf

BARBOSA, V.M.S.; SILVA, J.V.S .Utilização De Teorias De Enfermagem Na Sistematização Da Prática Clínica Do Enfermeiro: Revisão Integrativa. Rev. Enf. Atenção à Saúde, V. 7, n.1, 2018. [Citado em 15 Março 2021]. Disponível em: http://seer.uftm.edu.br/revistaeletronica/index.php/enfer/article/view/2517.

BRANDÃO, M.A.G ET AL. Teorias de enfermagem na ampliação conceitual de boas práticas de enfermagem. Rev. Bras. Enferm., Brasília , v. 72, n. 2, p. 577-581, abr. 2019 . [Citado em 15 Março 2021]. Disponível em: http://www.scielo.br/scielo.php?script=sci arttext\&pid=S0034-71672019000200577\&lng=pt $\underline{\text { \&nrm }=\text { iso. }}$.

BRYANT-LUKOSIUS, D. ET AL. Advanced Practice Nursing: A Strategy for Achieving Universal Health Coverage and Universal Access to Health. Rev. Latino-Am. Enfermagem, Ribeirão Preto, v. 25, e2826, 2017. [Citado em 15 Março 2021]. Disponível em: http://www.scielo.br/scielo.php?script=sci_arttext\&pid=S0104-11692017000100301\&lng=en $\&$ nrm=iso. 


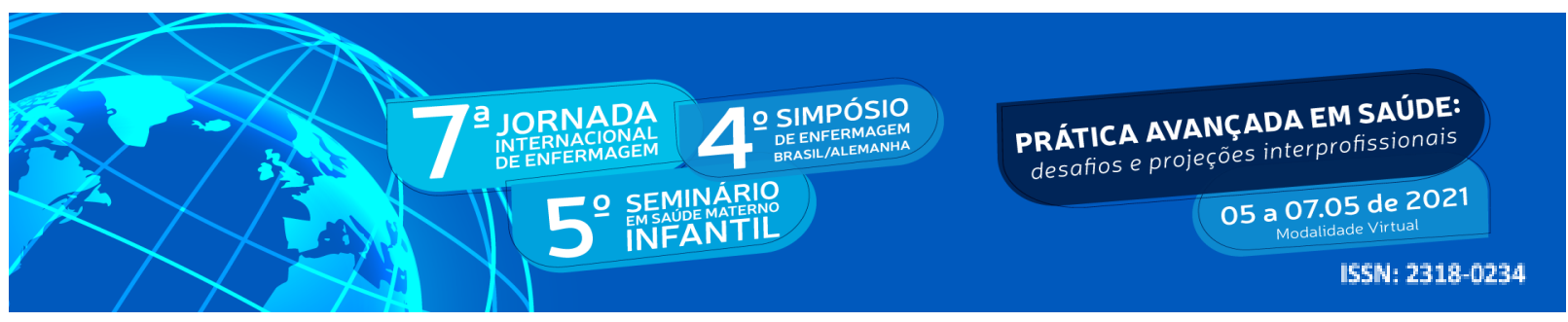

GOMES, R. M. ET AL. Sistematização Da Assistência De Enfermagem: Revisitando A Literatura Brasileira. Id on Line Rev. Mult. Psic. V.12, N. 40., 2018 [Citado em 15 Março 2021]. Disponível em: https://idonline.emnuvens.com.br/id/article/view/1167/1701.

INTERNATIONAL COUNCIL OF NURSES - CIE. Nurse practitioner/advanced practice nurse: definition and characteristics. ICN [Internet]. 2009 [Citado em 15 Março 2021]. Disponível em: https://acnp.org.au/sites/default/files/33/definition of apn-np.pdf

MARTINS, D. F.; BENITO, L. A. O. Florence Nightingale E As Suas Contribuições Para O Controle Das Infecções Hospitalares. Universitas: Ciências da Saúde, Brasília, v. 14, n. 2, p. 153-166, jul./dez. 2016 [Citado em 15 Março 2021]. Disponível em: https://www.publicacoesacademicas.uniceub.br/cienciasaude/article/view/3810.

MELEIS, A.I. Transitons theory: middle-range and situation-specific theories in nursing research and practice. New York: Springer; 2010.

MIRANDA NETO, M.V.M. ET AL. Advanced practice nursing: a possibility for Primary Health Care?. Rev Bras Enferm. 2018 [Citado em 15 Março 2021]. Disponível em: http://dx.doi.org/10.1590/0034-7167-2017-0672

PIMENTA, C. A. M. Enfermeiro de Prática Avançada: implementação no Brasil. 1st Oncology Nursing Brazil. 2018. [Citado em 15 Março 2021]. Disponível em: https://rvmais.iweventos.com.br/upload/cartas/files/10h00\%20Cibele $\% 20$ Andruccioli $\% 20-\%$ 20Cavalcanti\%2010-08.pdf

SILVA, M.C.N. ET AL. Protagonismo da Enfermagem Brasileira no combate ao COVID-19. Enfermagem em Foco. v.11, n. 1.; 2020 [Citado em 15 Março 2021]. Disponível em: http://revista.cofen.gov.br/index.php/enfermagem/article/view/4073/0.

SOUSA, P. A. F. Do conceito de Enfermagem de Prática Avançada à Enfermagem Avançada. Enfermagem avançada: um guia para a prática. Editora Sanar, 2016. [Citado em 15 Março 2021]. Disponível em https://www.sanarsaude.com/images/p/Enfermagem $\% 20$ Avan $\% \mathrm{C} 3 \% \mathrm{~A} 7 \mathrm{ada} \% 20-\% 20$ Trecho.p df

VITALI, M. M. ET AL. Prumo, Guyton e caminho: polissemia da saúde na perspectiva dos estudantes universitários. Rev. CEFAC, São Paulo, v. 21, n. 6, e5619, 2019 . [Citado em 15 Março 2021]. Disponível em: http://www.scielo.br/scielo.php?script=sci_arttext\&pid=S1516-18462019000600502\&lng=en $\& n r m=$ iso. 